\title{
RARE CONGENITAL ANOMALIES AT THE CRANIOVERTEBRAL JUNCTION; OS ODENTOIDEUM
}

\author{
Murat Guntel $^{1}$ and Hanifi Bayarogullari ${ }^{1}$ \\ ${ }^{1}$ Mustafa Kemal Universitesi Tayfur Ata Sokmen Tip Fakultesi
}

May 28, 2021

\begin{abstract}
Aim: We aimed to identify dislocation, myelomalacia, and other congenital pathologies that may cause neurological complications arising from the odontoid process in the craniocervical junction (CVJ) of patients who were performed cervical tomography due to various complaints. Materials and Methods: Patients who were performed cervical CT with various complaints between 2010 and 2018 were retrospectively screened and evaluated, from the hospital registry system. Segmentation and fusion anomalies arising from the odontoid process in the craniovertebral junction area, as well as other accompanying pathologies such as subluxation, dislocation, compression to the spinal cord, and myelomalacia were also recorded. An additional 1.5 Tesla cervical MRI was performed for the 12 patients with os odontoideum. Radiological evaluation was performed on the workstation by an experienced radiologist. Results: 2525 patients with cervical CT were included in the study. 67 of these patients had congenital anomalies at the CVJ. 13 of 67 patients had os odontoideum, 38 had Klippel-Feil syndrome (KFS). Atlanto-occipital assimilation (AOA) and KFS often saw together. Only AOA was observed in 16 cases. Neck pain and limitation of movement were the most common findings in patients with congenital anomalies in the CVJ region. Conclusion: Congenital anomalies at the CVJ region, although rare, can cause mortality or morbidity. Cervical CT and MRI studies on the CVJ region should be evaluated carefully by the clinicians due to the risk of various complications.
\end{abstract}

\section{RARE CONGENITAL ANOMALIES AT THE CRANIOVERTEBRAL JUNCTION; OS ODENTOIDEUM}

\begin{abstract}
Aim: We aimed to identify dislocation, myelomalacia, and other congenital pathologies that may cause neurological complications arising from the odontoid process in the craniocervical junction (CVJ) of patients who were performed cervical tomography due to various complaints.

Materials and Methods: Patients who were performed cervical CT with various complaints between 2010 and 2018 were retrospectively screened and evaluated, from the hospital registry system. Segmentation and fusion anomalies arising from the odontoid process in the craniovertebral junction area, as well as other accompanying pathologies such as subluxation, dislocation, compression to the spinal cord, and myelomalacia were also recorded. An additional 1.5 Tesla cervical MRI was performed for the 12 patients with os odontoideum. Radiological evaluation was performed on the workstation by an experienced radiologist.

Results: 2525 patients with cervical CT were included in the study. 67 of these patients had congenital anomalies at the CVJ. 13 of 67 patients had os odontoideum, 38 had Klippel-Feil syndrome (KFS). Atlantooccipital assimilation (AOA) and KFS often saw together. Only AOA was observed in 16 cases. Neck pain and limitation of movement were the most common findings in patients with congenital anomalies in the CVJ region.
\end{abstract}

Conclusion: Congenital anomalies at the CVJ region, although rare, can cause mortality or morbidity. 
Cervical CT and MRI studies on the CVJ region should be evaluated carefully by the clinicians due to the risk of various complications.

Keywords: Craniovertebral junction; Os Odentoideum; Multislice Computed Tomography; Magnetic Resonance Imaging; Complications

\section{What's already known about this topic?}

Craniovertebral junction is a junction with a complex structure and numerous congenital or acquired (traumatic, inflammatory) pathologies can affect this anatomic region.

There are many studies in the literature that emphasize this anatomical structure and underline that its anomalies may cause mortality or morbidity.

\section{What does this article add?}

With this study, 2525 cervical CT scans obtained for various reasons between 2010 and 2018 were scanned and we estimate that it is one of the largest series researched in the literature.

Clinicians and radiologists should pay particular attention to the evaluation of this anatomical region.

\section{Introduction}

Craniovertebral junction (CVJ) is a junction with a complex structure consisting of ligaments providing stability for the bones and joints between the head and spinal column. Bony structures in this junction are occipital bone (1) including the foramen magnum, clivus, and condylar process and the first two vertebrae of the cervical spinal column: atlas $(\mathrm{C} 1)$ and axis $(\mathrm{C} 2)$. Atlanto-occipital joint (AOJ), atlanto-dental joint $(\mathrm{ADJ})$, and atlanto-axial joint $(\mathrm{AAJ})$ are present in this complex structure. There are multiple ligaments and membranes providing stability on the joint structures, however, the most important of those are cruciate and alar ligaments. $\mathrm{C} 1$ and $\mathrm{C} 2$ vertebrae together with the other joints in this region help to move the head. While AOJ is responsible for the anterior and posterior movement of the head, ADJ is responsible primarily for the axial rotation of the head. Cranio-cervical junction (CCJ) is the region where the brainstem, spinal cord, some cranial nerves, and vertebral arteries are located and preserved. Numerous congenital or acquired (traumatic, inflammatory) pathologies can affect the CVJ. Among the congenital pathologies, atlanto-occipital assimilation (AOA), Klippel-Feil syndrome (KFS), and similar rare segmentation and ossification abnormalities, malformations like Os Odentoideum (OO) can narrow the spinal canal and can lead to myelomalacia and neurological findings by compressing the spinal cord (1-3).

In this study, we aimed to present the patients who was performed cervical tomography due to various complaints and had congenital pathologies originated from odontoid proses in the CVJ region causing dislocation, myelomalacia, and neurological complications.

\section{Materials and methods}

\section{Patients and imaging procedures}

2525 patients for whom cervical Multislice Computed Tomography (MSCT) was performed due to head and neck pain, various neurological complaints, trauma, etc. between 2010 and 2018 were evaluated for segmentation and fusion anomalies of odontoid process in the CVJ region. Patients with coexisting pathologies such as subluxation, dislocation, compression to the spinal cord, and myelomalacia (MM) were included in the study as well. Toshiba Aquilon 64 MDCT device was used for the imaging studies. An additional 1.5 Tesla MRI (Achieva, Philips, Best, The Netherlands) was performed for the 12 patients with os odontoideum. Radiological evaluation was performed on the workstation by an experienced radiologist.

Ethical approval was obtained from Mustafa Kemal University Ethical council (Approval no: 04, dated 03.12.2020).

\section{Results}


1690 of the patients for whom MSCT was performed were male and 835 were female. 67 of these patients had congenital anomalies at the CVJ. 13 of 67 patients had os odontoideum (OO), 38 had KFS, AOA was also frequently observed in KFS. Only AOA was observed in 16 cases. KFS and AOA often saw together. 10 patients with $\mathrm{OO}$ were male and 3 were female. The mean age of the patients with OO was 43.07 (range: 14-88). 22 patients with KFS were male and 16 were female. The mean age of these patients was 41.68 (range:0-71). 10 patients with AOA were male and 6 were female. The mean age of these patients was 39.12 (range:10-70) (Table 1).

Neck pain and limitation in the range of motion around the neck region were the most frequent finding in the patients who had congenital anomalies at the CVJ region. Various neurological findings were also present in these patients in accordance with the severity of coexisting dislocation and degenerative changes. Additionally, atlanto-occipital dislocation (AOD), Atlanta-dental dislocation (ADD), and atlanto-axial dislocation (AAD) were also detected. Loss of muscle strength and paralysis were observed clinically especially in the patients with MM who had prominent nerve compression.

9 of 13 patients with OO had dystopic (Figure 1a-b) and 4 were orthotopic type. 1 patient with OO had coexisting AC, 4 had AOD, 3 had ADD, 2 had roto-AAD, and 5 had MM. Fusion with the anterior arch of $\mathrm{C} 1$ vertebra in 1 patient with $\mathrm{OO}$ (Figure 1c-e) and fusion with clivus in another patient with OO was observed. 1 patient with OO had coexisting AOA (Table 1).

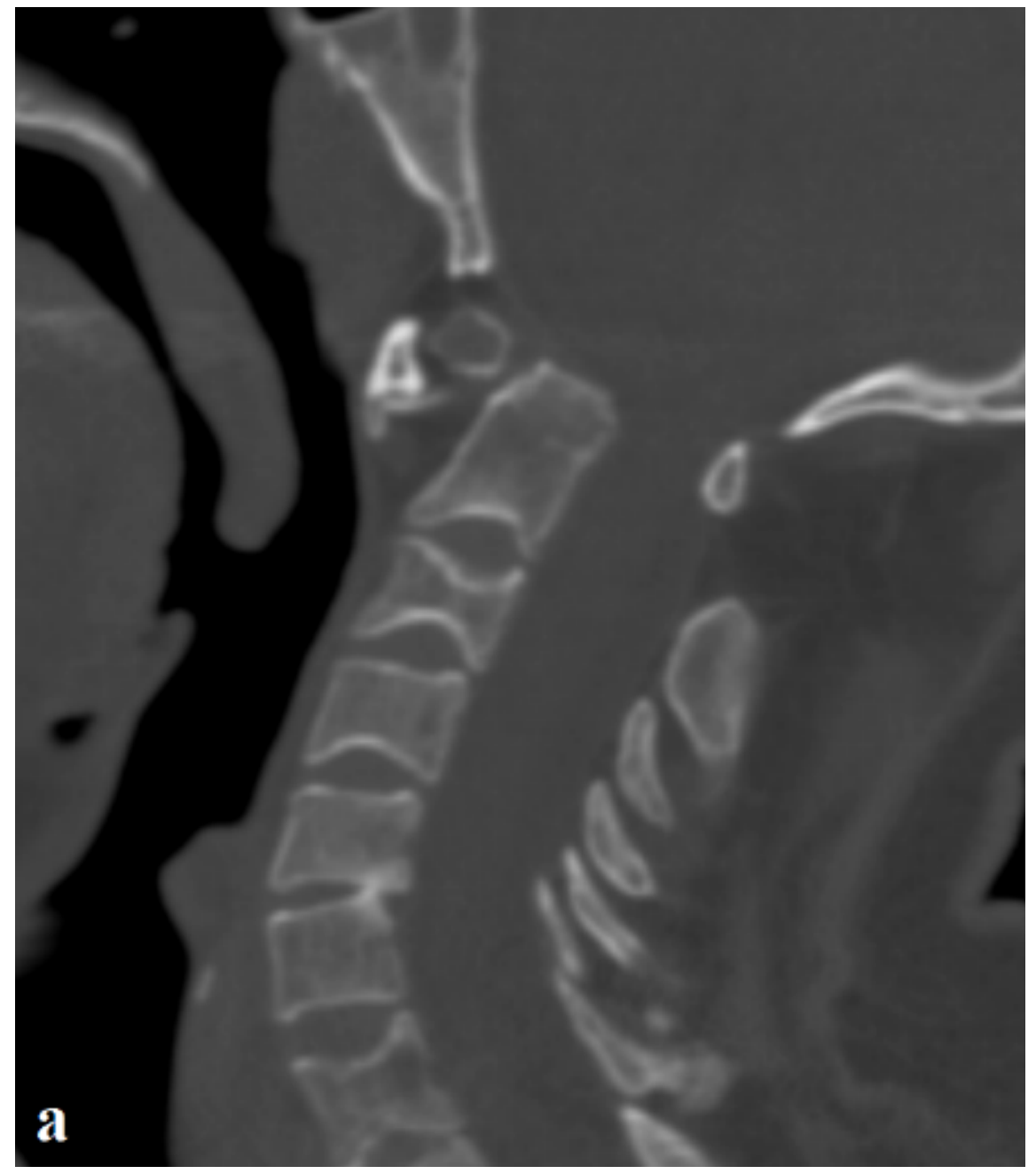




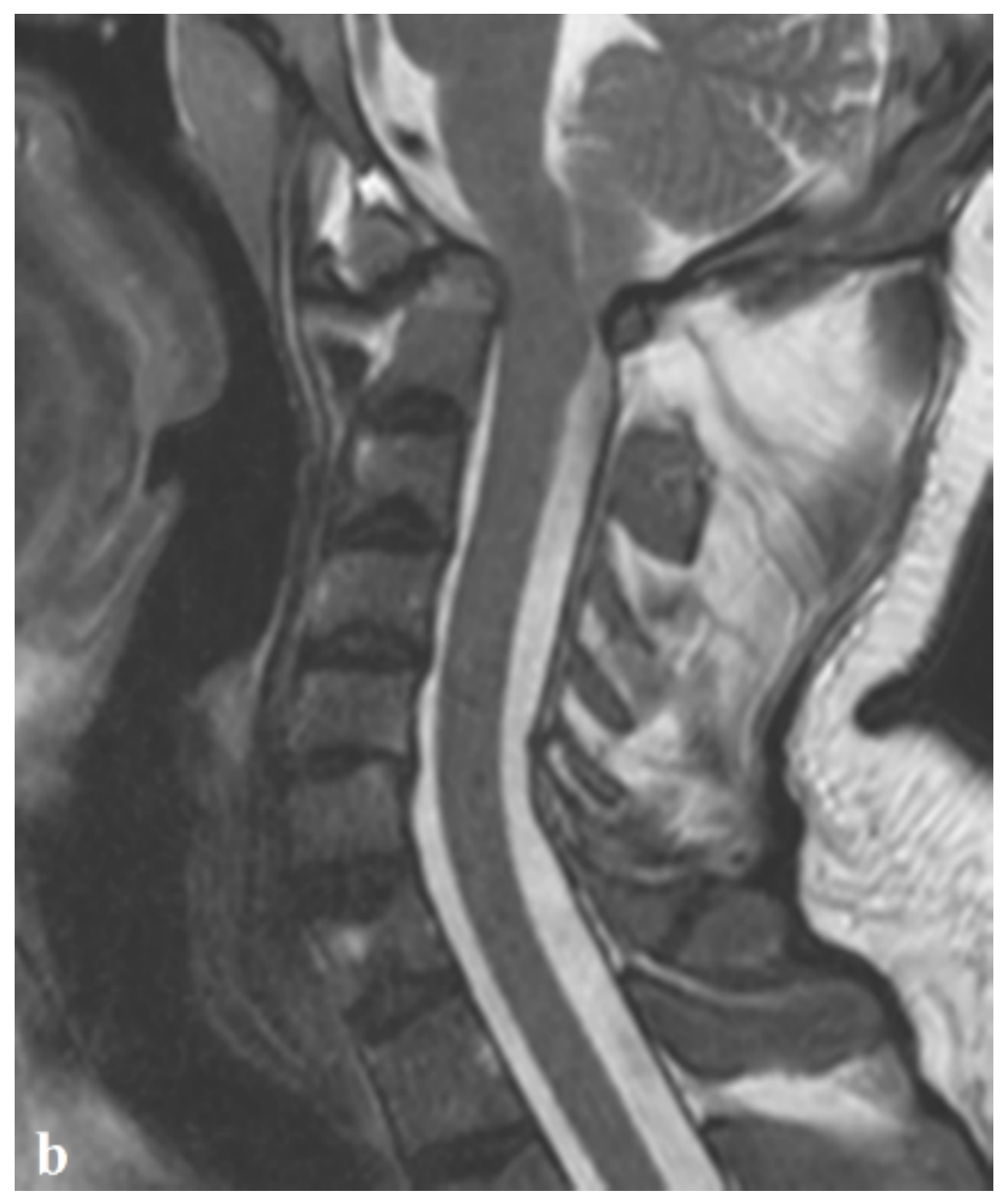

Figure 1a Figure 1b 


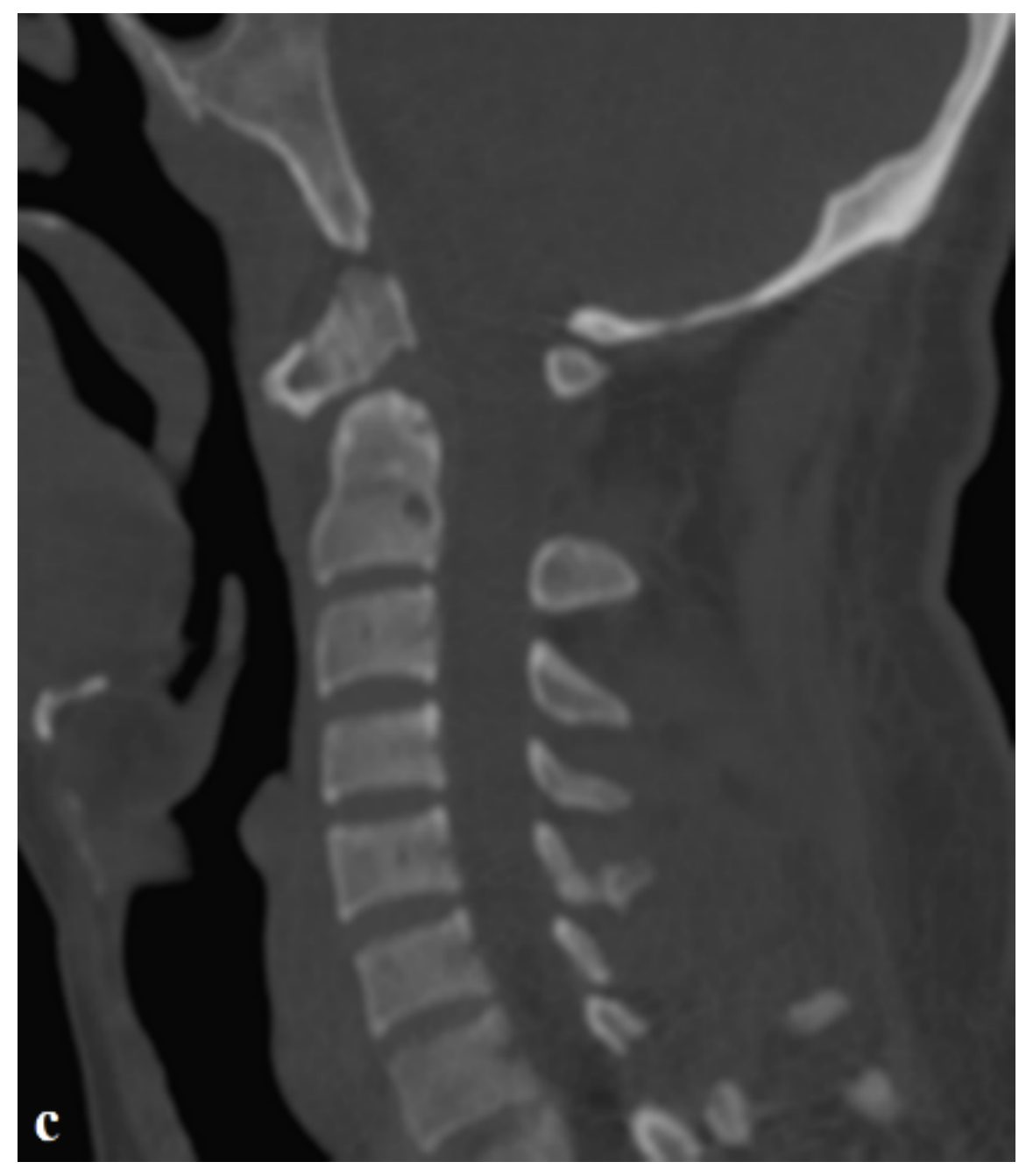




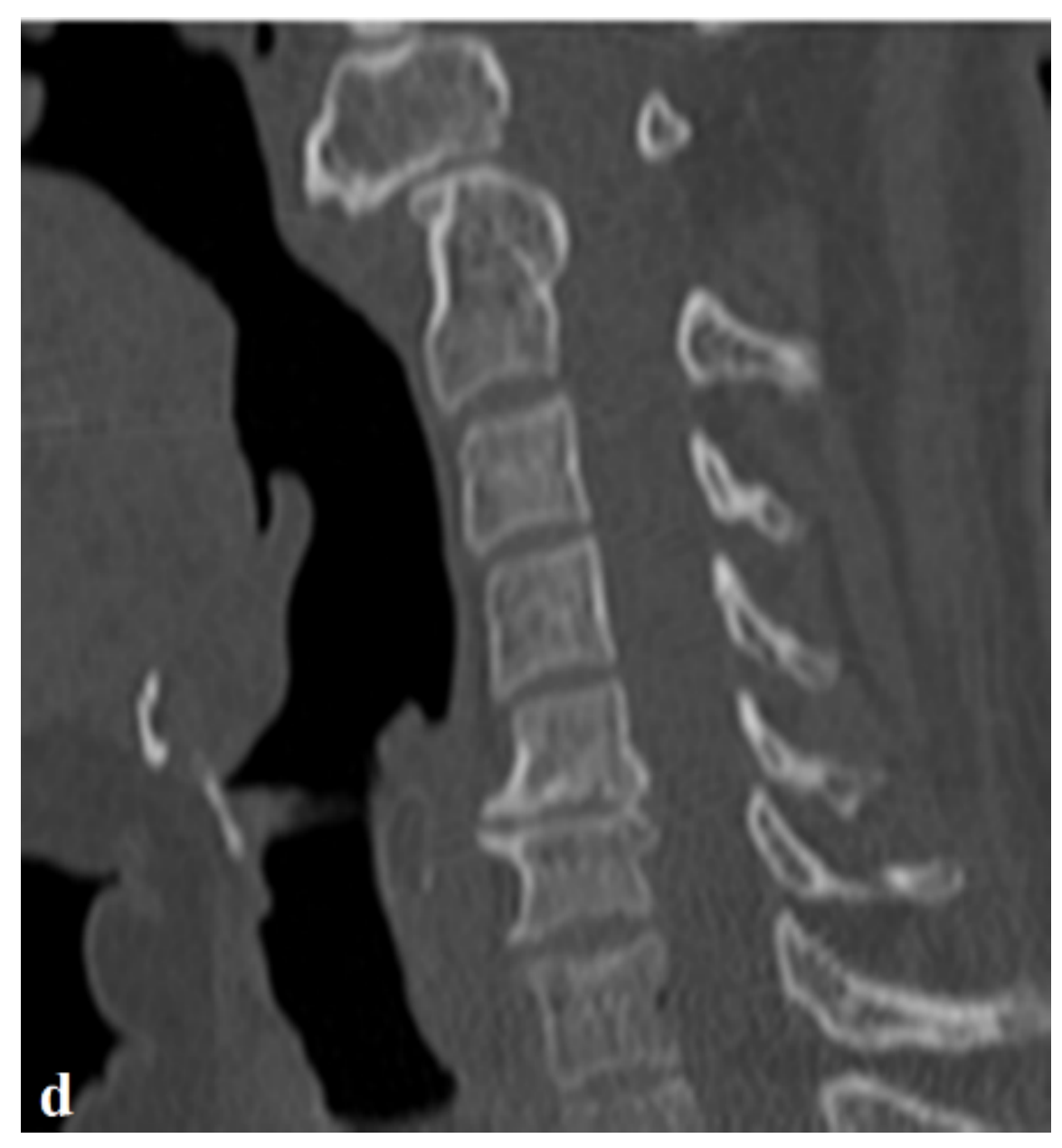




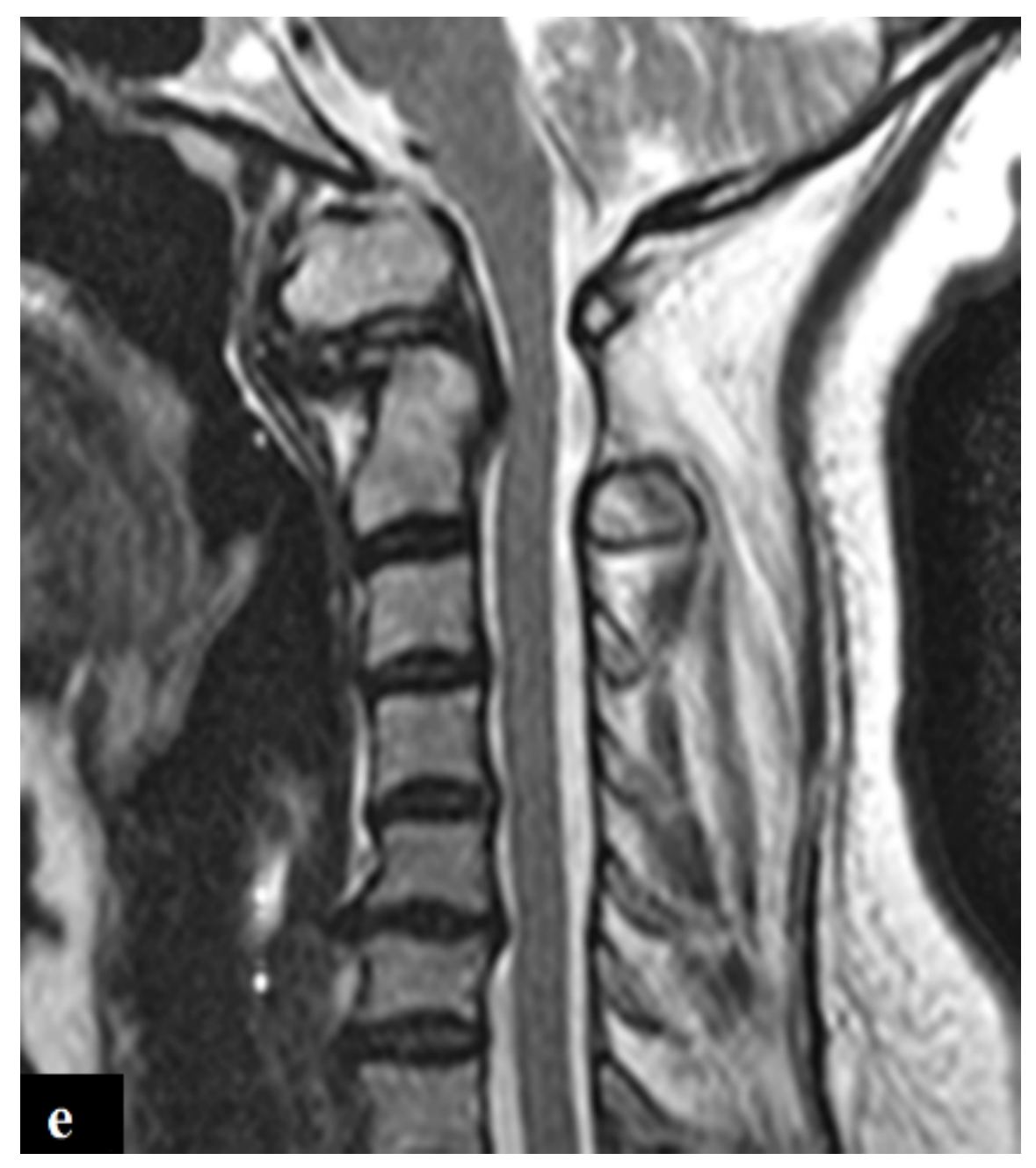

Figure 1c Figure 1d Figure 1e

Fusion between the $\mathrm{C} 1$ and $\mathrm{C} 2$ vertebrae in 2 patients with KFS and fusion between only $\mathrm{C} 2$ and $\mathrm{C} 3$ vertebrae in the remaining 36 cases with KFS were observed 21 patients with KFS had bilateral AOA, 1 had left partial AOA, 10 had odontoid hypoplasia (OH), 11 had basilar invagination (BI), 6 had Arnold Chiari (AC), 1 had AOD, 9 had ADD, 3 had rotatory AAD, 5 had MM, and 4 had coexisting syringomyelia (Figure 2 a-e). 15 patients with KFS had a posterior arch defect in the C1 vertebra and 4 had an anterior arch defect (Table $1)$. 


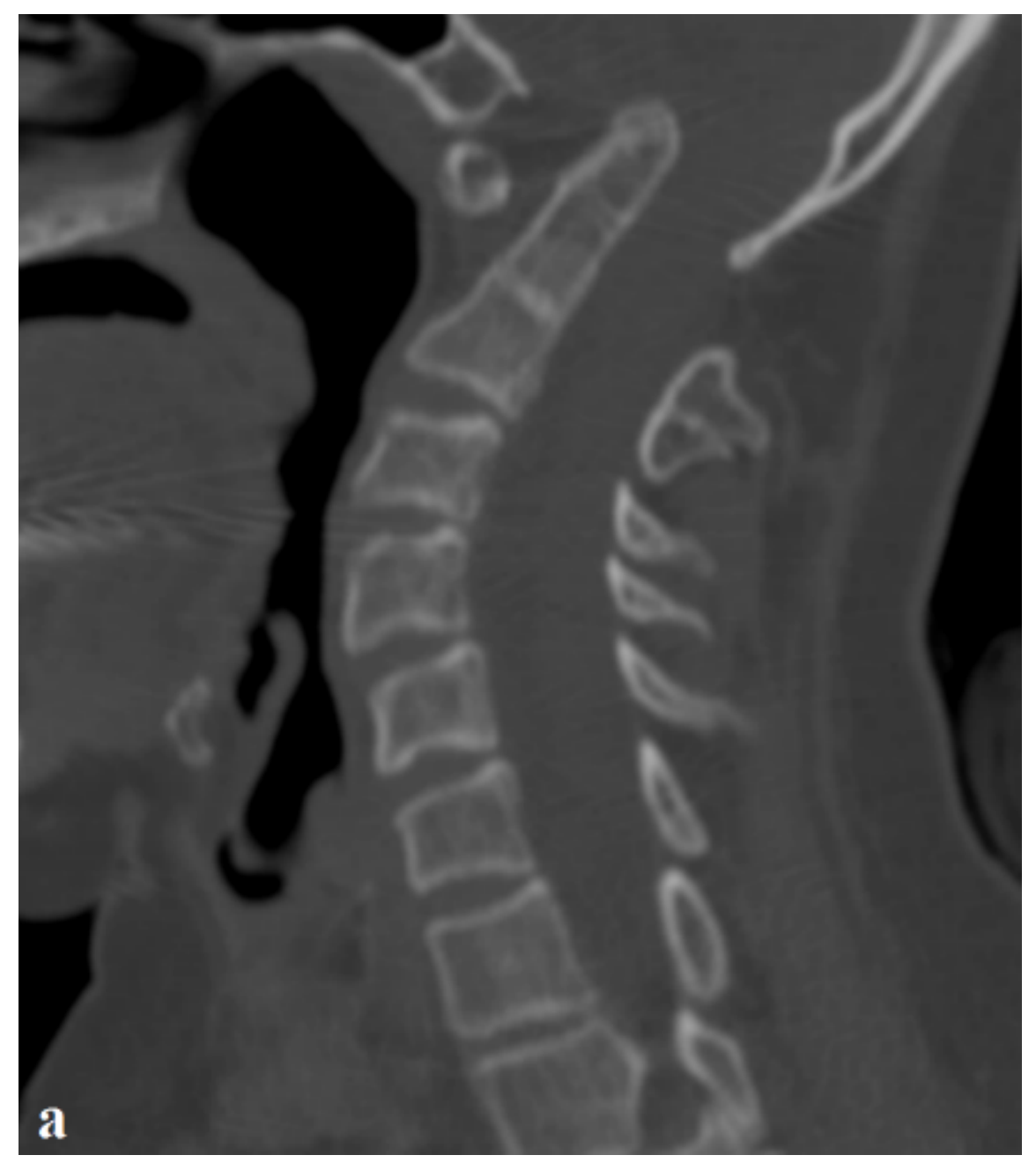




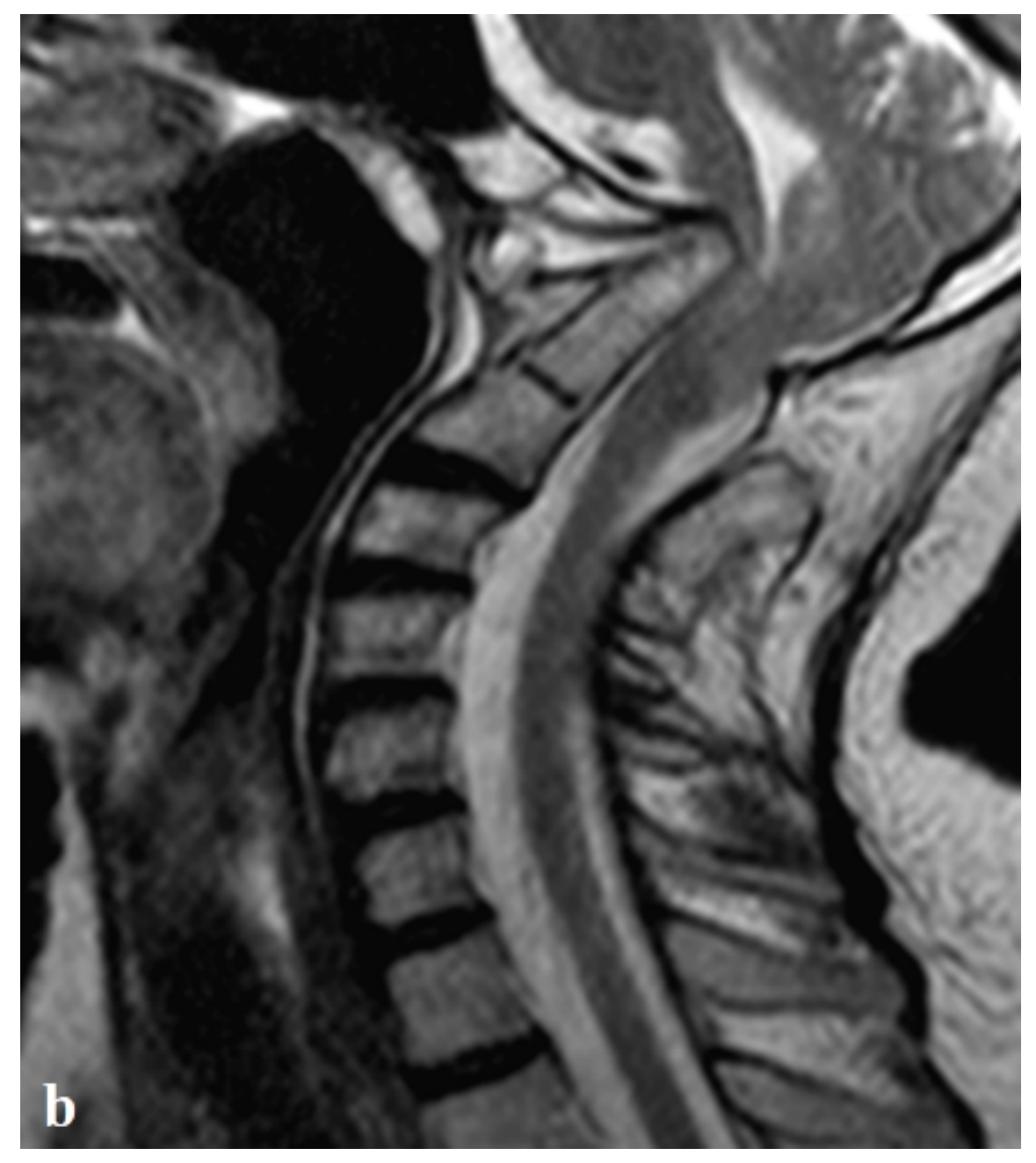




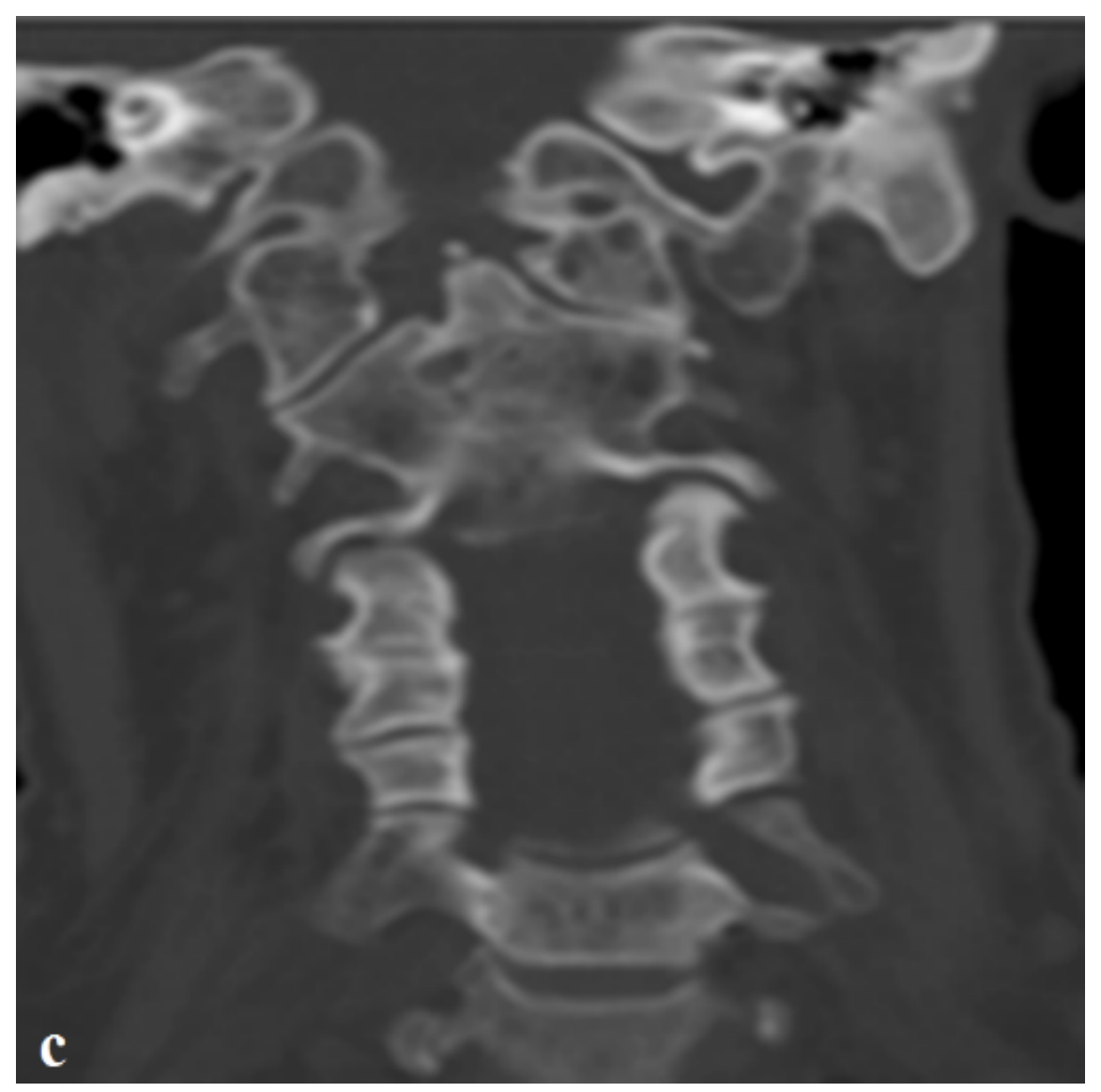

Figure 2a Figure 2b Figure 2c 


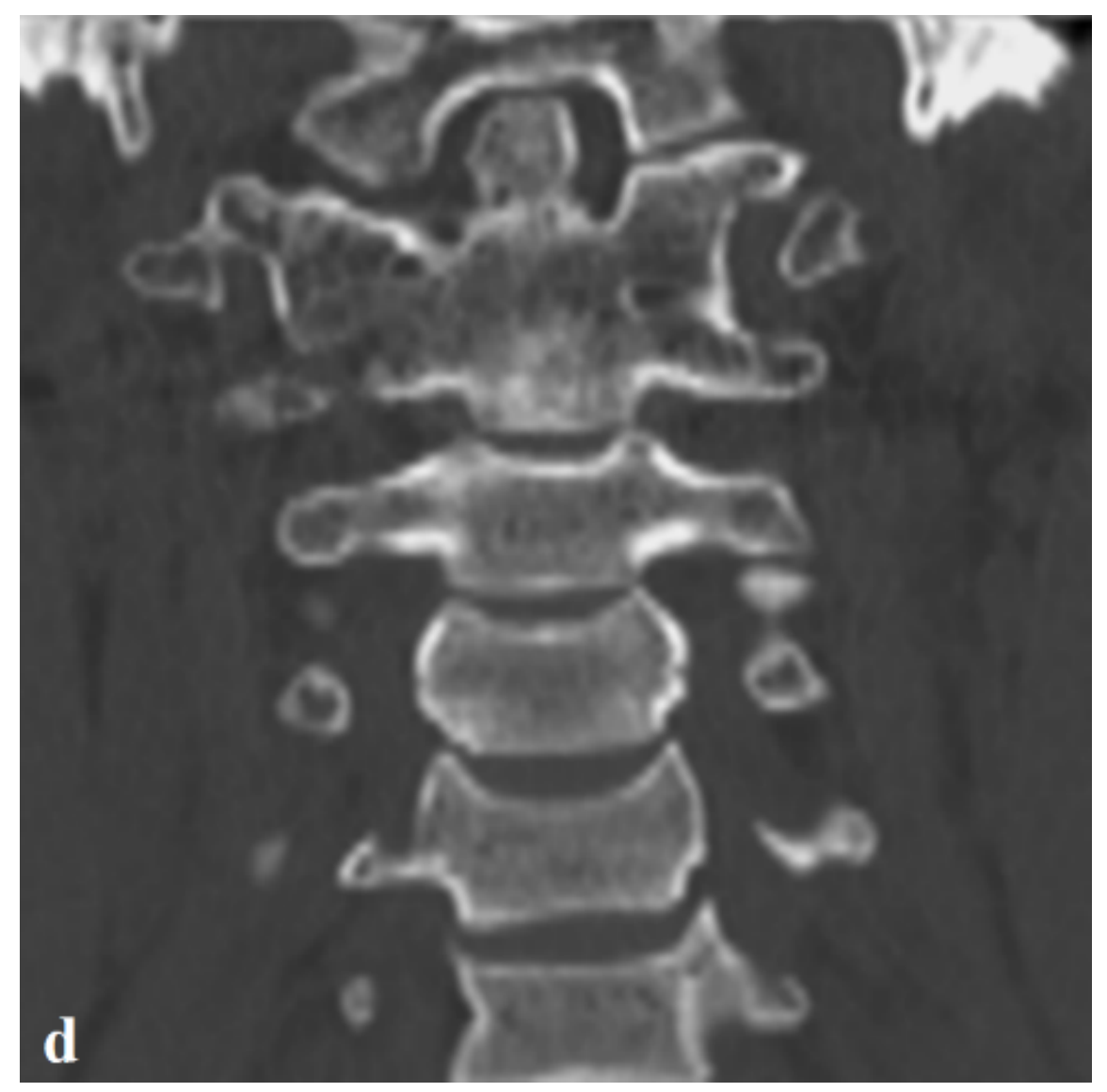




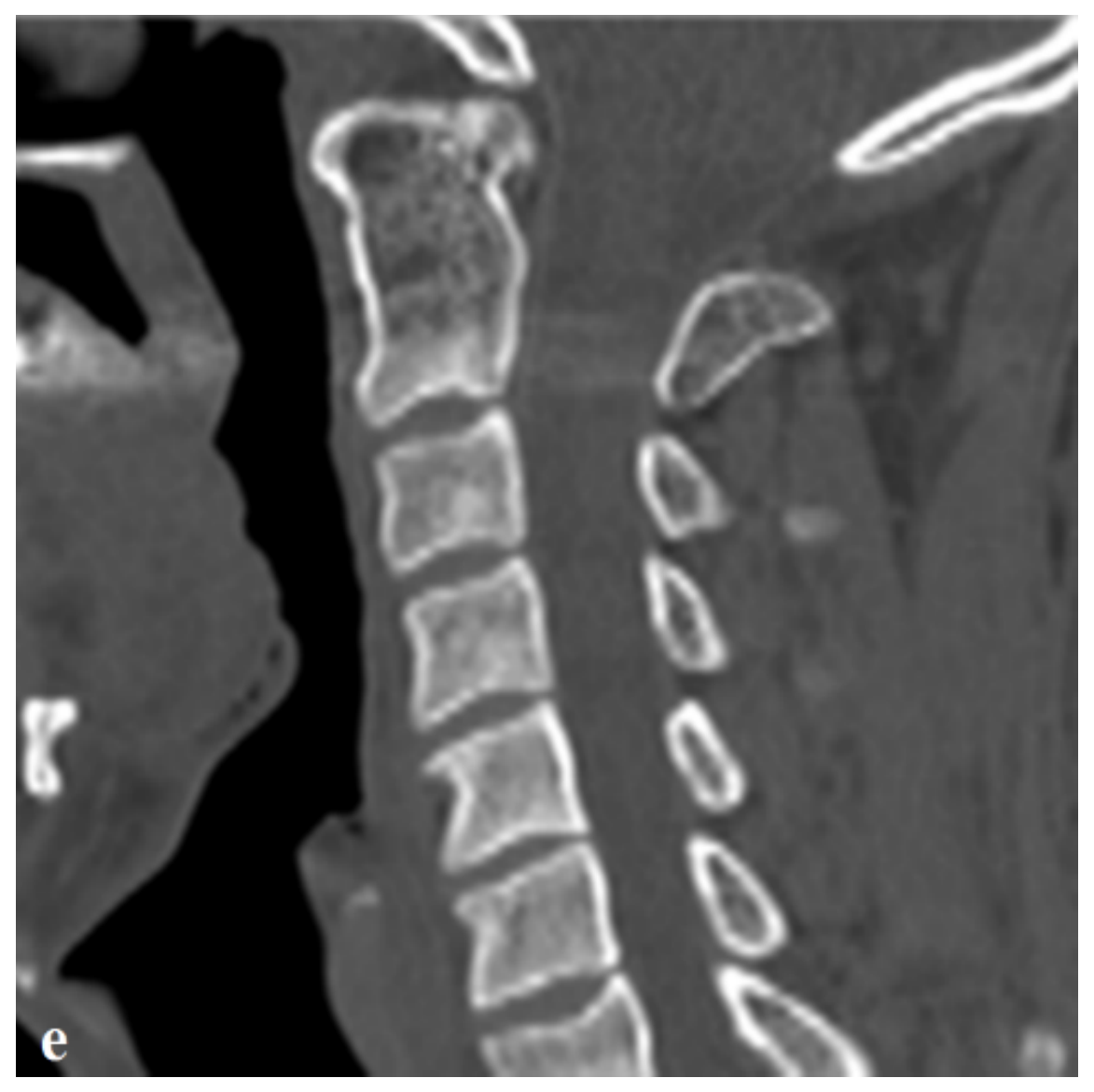

Figure 2d Figure 2e

1 patient with AOA had partial (right) and 15 patients had total assimilation. 4 patients with AOA had $\mathrm{OH}, 3$ had BI, 5 had AC, 1 had ADD, 2 had rotatory AAD, and 3 had MM. 3 patients with AOA had a posterior arch defect in the $\mathrm{C} 1$ vertebra (Figure 3 a-c), (Table 1). 


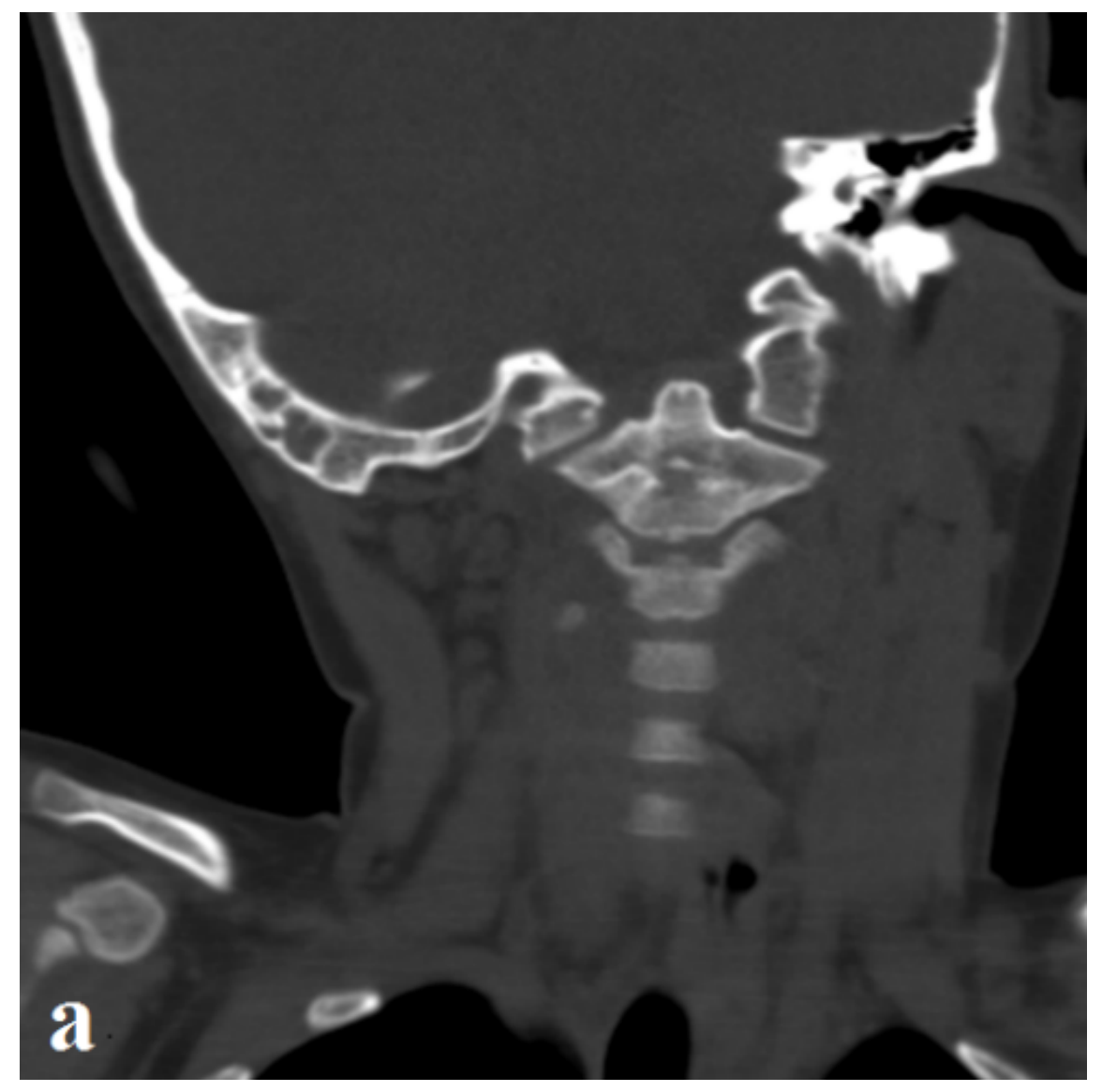




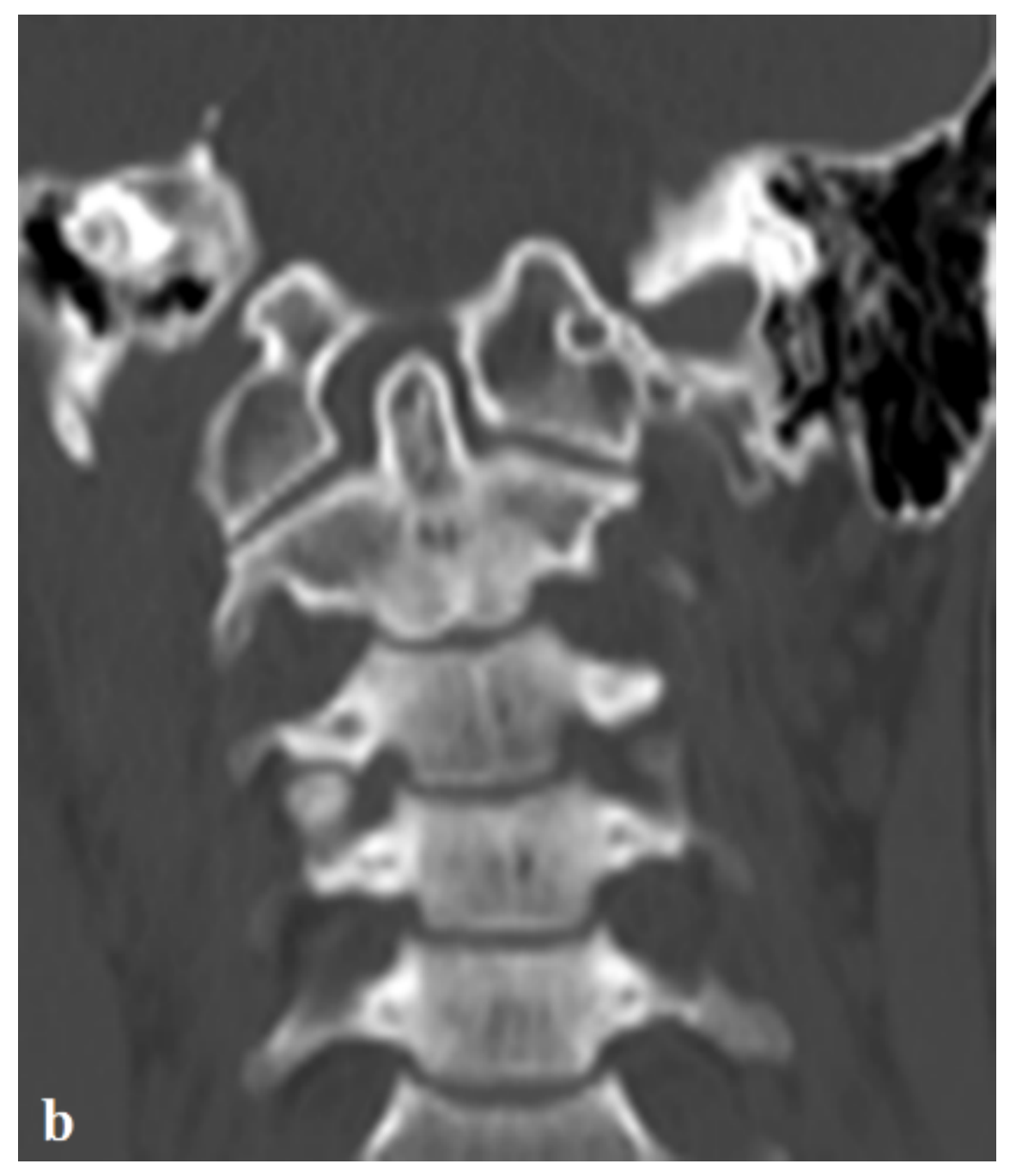




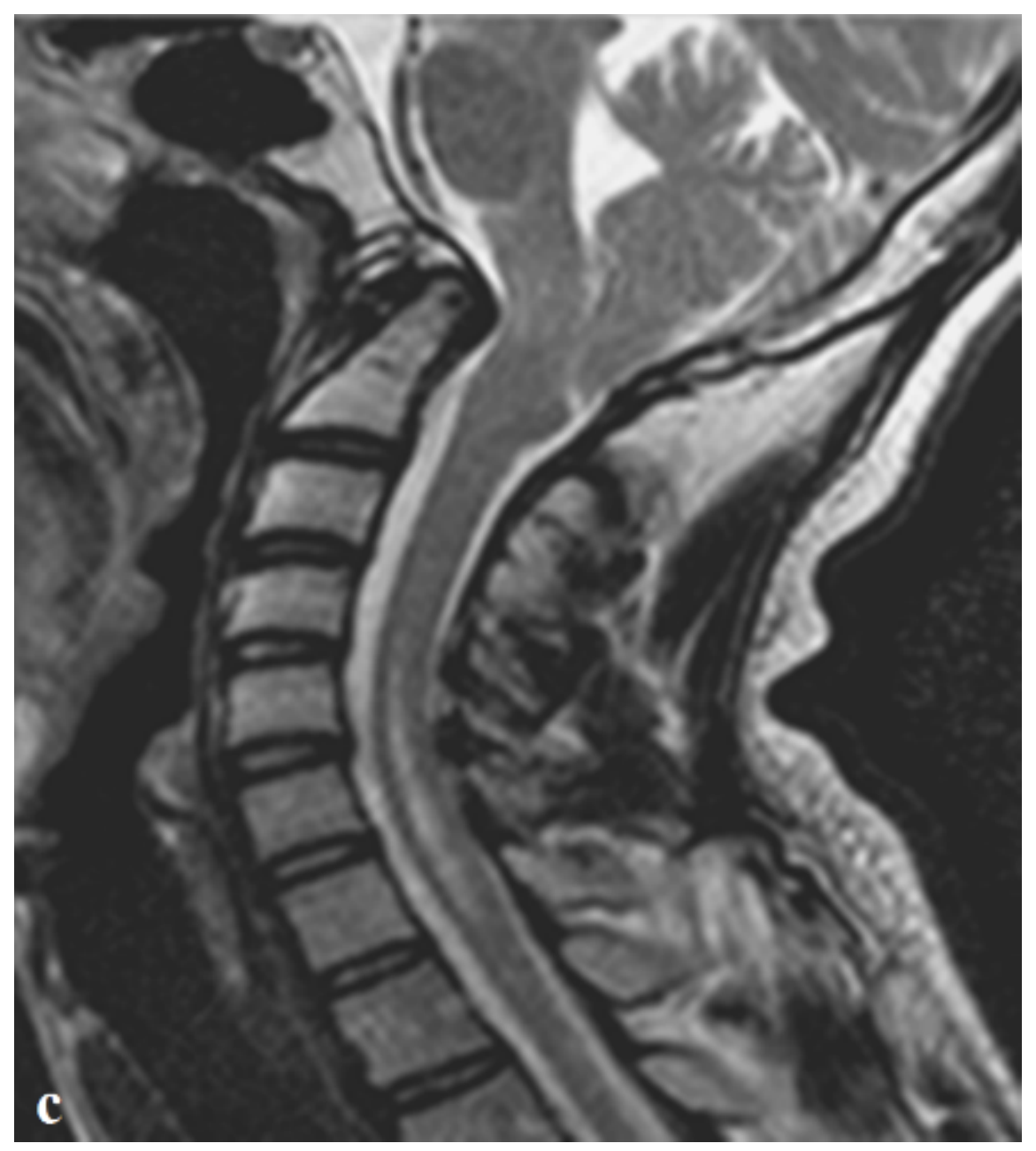

Figure 3a Figure 3b Figure 3c

While the mean atlanto-dental instability of ADD was $5.48 \mathrm{~mm}$ (range 3.5-10.4 mm) in the patients with KFS, it was $6.56 \mathrm{~mm}$ (range: $3-8.8 \mathrm{~mm}$ ) in the patients with AOA. While the mean dislocation of dens towards the cranial side in the patients with KFS and basilar invagination (BI) was 10.86 (range:7.7-15.29 $\mathrm{mm}$ ), it was 10.07 (range:6.1-15.5 mm) in the patients with AOA and BI (Table 1).

Table 1.

\begin{tabular}{|c|c|c|c|c|c|c|c|c|c|c|c|c|c|c|}
\hline & Num. & Gender & Gender & Age & Age & Age & $\mathrm{OH}$ & $\mathrm{BI}$ & $\mathrm{AC}$ & AOD & ADD & RAAD & MM & $\mathrm{Sy}$ \\
\hline OO & 13 & $\begin{array}{l}\text { Male } \\
10\end{array}$ & $\begin{array}{l}\text { Female } \\
3\end{array}$ & $\begin{array}{l}\text { Min. } \\
14\end{array}$ & $\begin{array}{l}\text { Mid. } \\
\text { 43.07 }\end{array}$ & $\begin{array}{l}\text { Max. } \\
88\end{array}$ & - & - & 1 & 4 & 3 & 2 & 5 & - \\
\hline AOA & 16 & 10 & 6 & 10 & 39,12 & 70 & 4 & 3 & 5 & - & 1 & 2 & 3 & - \\
\hline KFS & 38 & 22 & 16 & 0 & 41,68 & 71 & 10 & 11 & 6 & 1 & 9 & 3 & 5 & 4 \\
\hline
\end{tabular}

Abbreviation; OO (os odontoideum), AOA (atlanto-occipital assimilation), KFS (Klippel-Feil syndrome), Num (number), OH (odontoid hypoplasia), BI (basilar invagination), AC (Arnold Chiari), AOD (Atlantooccipital dislocation), ADD (atlanto-dental dislocation), RAAD (rotatory atlantoaxial dislocation), MM (myelomalacia), Syr. (syringomyelia) 


\section{Discussion}

The structures forming the CVJ are originated embryologically from the $4^{\text {th }}$ occipital and $1^{\text {st }}$ and $2^{\text {nd }}$ cervical sclerotomes. Occipital condyles, the superior part of the posterior arch of the atlas, and apex of the dens are originated from the $4^{\text {th }}$ occipital sclerotome (proatlas). The inferior part of the posterior arch of the atlas, anterior arch of the atlas, and dens are originated from the $1^{\text {st }}$ cervical sclerotome and the body of the axis is originated from the $2^{\text {nd }}$ cervical sclerotome. Congenital anomalies in the CVJ develop secondary to the segmentation abnormalities in these sclerotomes. These anomalies can be in the form of fusion as the fusion of two different structures or development or formation abnormalities of the structures leading to the dislocation or subluxation of the joints $(4,5)$.

Secondary to the segmentation anomalies in the $4^{\text {th }}$ occipital sclerotome and $1^{\text {st }}$ and $2^{\text {nd }}$ cervical sclerotomes, AOA, KFS, os odontoideum, and the other odontoid malformations and structural congenital anomalies such as BI and AC that can develop secondary to and/or independent from the former anomalies can be observed together. Instability in the joints at the CVJ region, changes in the ligament laxity, joint dislocations, early degenerative changes in the bony structures can be observed secondary to these congenital malformations. Joint dislocations and degenerative changes secondary to the congenital malformations can lead to a narrowing at the CVJ region and compression to the medulla spinalis, cranial nerves, and vascular structures and consequent various neurological findings, severe neurological problems, morbidity, and mortality $(6,7)$.

Among the CVJ malformations, AOA was described in 1884 by Rokitansky. It is observed in $0.12-0.72 \%$ of the population with no gender predilection. Atlas fused with occipital bone acts as a functional unit of the occipital bone. Assimilation can form partially or completely. Foramen magnum narrowing, basilar invagination, and atlanto-axial instability are observed with this variation $(8,9)$. In our study, 16 isolated AOA cases and 22 AOA cases with KFS were detected within 8 years. Among these 38 AOA cases, 2 were partial AOA and 36 were bilateral AOA cases. The rate of isolated AOA was $0.63 \%$ and the rate of coexisting KFS was 1.5\%. Among the cases with isolated AOA, 5 had AC, 3 had BI, 4 had OH, 2 had RAAD, 1 had $\mathrm{ADD}$, and 3 had MM.

KFS is one of the most frequent segmentation anomalies that develops secondary to the segmentation abnormalities in the cervical sclerotomes in the early gestational period and was described as the fusion of two or more cervical vertebrae. It is described firstly by Maurice Klippel and Andre Feil in 1912. It is observed in 1 of each 40000 births and the male/female ratio was reported as 3/2. Short neck, low dorsal hairline, and restricted neck mobility triad can be observed in $50 \%$ of the patients with KFS. Other system pathologies including spinal anomalies such as scoliosis and kyphosis, renal anomalies, Sprengel deformity, deafness, facial asymmetry, and congenital cardiac disorders can be observed together with this syndrome. It is divided into three groups by Feil as the prominent fusion of the cervical vertebra, type I; cervical fusion at one or two levels, type II; the presence of concomitant thoracic and lumbar anomalies, type III. The most observed anomaly at the CVJ is the fusion of $\mathrm{C} 2$ and $\mathrm{C} 3$ leading to increased mobility of this region causing more frequent dislocations and thus leading to neck pain and severe neurological findings due to its spinal cord compression $(10,11)$. The rate of KFS was $1.5 \%$ in our study and male patients were more frequently affected. In our study, 2 patients had fusion at the C1-2 level and 36 patients had fusion at the C2-3 level (type 2 KFS). Among the KFS cases, 22 had AOA, 10 had OH, 11 had BI, 6 had AC. Among the 13 KFS cases with dislocation, 9 were ADD, 3 were RAAD, and 1 was AOD. Among the KFS cases, 5 had MM and 4 had a syrinx cavity.

Odontoid and atlas malformations are the anomalies at the CVJ causing dislocations secondary to the joint instabilities. Therefore, it is important to know the ossification and synchondrosis centers of the atlas at the prenatal and postnatal periods. The odontoid process can be distinguished from the axis body with a cartilaginous band, dentocentralsynchondrosis. This area closes at 3 years of age. If it does not close, os odontoideum (OO) develops. The odontoid process develops from the caudal half of the first cervical sclerotome and from the proatlas that develops from its cranial half $(1,2)$. OO is a rare anomaly at the CVJ region that is located on the anterior arch of the atlas with a smooth cortical surface and it is branched from 
the main body of the odontoid process. It is described firstly by Giacomini in 1866. Despite controversial etiology, it was linked to the congenital reasons related to the absence of fusion at the synchondrosis level or the acquired reasons related to the absence of fusion at the synchondrosis level due to trauma-related fractures. $\mathrm{OO}$ was divided into two groups as orthotopic and dystopic by Fielding. It is named as orthotopic type in case of its normal localization at the posterior of the anterior arch of the atlas and if it is located in another position it is named as dystopic type. Its dystopic type can mimic a type II dens fracture. Dystopic type is observed as a separate bone structure with varying sizes. The lower limit of the OO is accepted as its location above the superior facet of the axis and half of the size of a normal odontoid process.OO is observed more frequently in the $2^{\text {nd }}$ and $3^{\text {rd }}$ decade with a male predominance. It can be observed asymptomatically as well as it can cause secondary neurological and vascular problems due to compression to the cervicomedullary structure and vertebral artery related to AAD which develops secondary to the mobile aberrant bone structure and the insufficiency of the cruciate ligament. Some OO cases can be observed together with BI. Neck pain, torticollis, myelopathy, and syncope can be observed as clinical findings $(12,13)$. In our study, the rate of $\mathrm{OO}$ was $0.51 \%$ and it was observed more frequently in male patients. Dislocation was observed in 11 of the 13 patients; 5 of them were ADD, 4 were AOD, and 2 were RAAD. 1 patient had concomitant $\mathrm{AC}$ and 5 had MM.

Os terminale (OT), Condylus Tertius, and $\mathrm{OH}$ are the other malformations of the odontoid process. OT develops secondary to the inability of the secondary ossification center formed in the odontoid process to join the structure of the odontoid process until 12 years of age. Despite low clinical importance, it can be confused with type 1 odontoid fracture. Condylus Tertius develops when the proatlas fuses wrongly with the occipital sclerotome that will give rise to clivus during the embryological development. Then, Condylus Tertius develops to form the $3^{\text {rd }}$ joint with the atlas and it is named as $3^{\text {rd }}$ occipital condyle. This structure can sometimes cause neural compression. $\mathrm{OH}$ is maldevelopment of the odontoid causing it to stay short. Its agenesis is very infrequent and leads to subluxation by compromising the stability of the atlanto-axial joint $(1,13,14)$. In our study, 3 cases with OT were detected. Additionally, $14 \mathrm{OH}$ cases with concomitant KFS or AOA were detected.

BI, platybasia, and AC can develop embryologically together with the other congenital anomalies or secondary to these anomalies. BI is the displacement of the cervical spinal column towards the skull base and two types of BI were described. Displacement of the dens into the foramen magnum was described as the type $1 \mathrm{BI}$ and secondary to this displacement, atlanto-axial instability is observed. Displacement of the odontoid bone towards the skull base without displacement into the foramen magnum was described as type 2 BI. Atlanto-axial instability is not observed in this type of BI (15). Craniometric measurements such as the Chamberlain line and McGregor line are used for the detection of BI and the most superior point of the odontoid process must be 5 and $7 \mathrm{~mm}$ above these lines, respectively. AC is a CVJ anomaly that develops secondary to the displacement of the posterior fossa structures such as cerebellar tonsils and brainstem towards the foramen magnum and upper spinal canal. 4 types of AC malformations have been described and type 1 is the simplest and the most commonly observed type in adult patients. It is described as over $5 \mathrm{~mm}$ herniation of the cerebellar tonsils below the foramen magnum. Type 2 and 3 were observed more frequently in child patients. The clinical findings, especially neurological findings in the BI and AC can vary according to the compression at the CVJ level, narrowing in the spinal canal, and compression to the medullospinal region or concomitant congenital anomalies such as syringomyelia $(16,17)$. 14 cases with BI were observed in our study and 3 of these patients had AOA, 11 had KFS. 12 cases with AC were observed in our study and 6 had concomitant KFS, 5 had AOA, and 1 had OO.

Instabilities and dislocations are observed in the joint structures secondary to the laxity in the ligaments and malformations in the bony structures due to congenital anomalies in the CVJ region. Dislocations at the AOJ, ADJ, and AAJ levels narrow the spinal canal and lead to several clinical findings secondary to the compression of the neural structures. The classification systems used for the joint dislocations are as follows: Traynelis classification for the AOJ dislocation, the atlantodental interval for the ADJ, and Fielding and Hawkins classification for the AAJ. While most of the AOJ dislocations develop after a high energy trauma, a limited number of nontraumatic cases were reported in the literature. Congenital dislocations 
and luxations at the CVJ are observed at the ADJ and AAJ. Congenital dislocations are not detected in the childhood period, rather they are detected after the $2^{\text {nd }}$ and $3^{\text {rd }}$ decade due to more frequent ligament laxity and joint dislocation at these ages $(18,19)$. ADD is defined as above $3 \mathrm{~mm}$ of atlanto-dental interval at the childhood period and above $5 \mathrm{~mm}$ of atlanto-dental interval at the adults. While Greenberg and Wang classifications are used to direct the management of the dislocations, Fielding and Hawkins classification are utilized for determining the way of dislocation. While dislocations are divided into two groups as correctable and uncorrectable in the Greenberg classification, Wang classification added 2 new parameters, instability and bone dislocation, based on the Greenberg classification. Treatment protocols are generated according to these classifications (20). The joint dislocations are observed more frequently at the advancing ages consistent with the previous reports and the most frequently observed dislocation was ADD. 9 cases with KFS, 5 cases with OO, and 1 case with AOA had concomitant ADD. 7 cases with RAAD were observed and 3 of them had KFS, 2 had AOA, and another 2 had OO. AOD was observed in 5 cases and 4 of them had concomitant $\mathrm{OO}$ and 1 had KFS.

This study includes some limitations. Firstly, the study was conducted retrospectively by investigation of the medical records of the patients. Secondly, because some patients were referred to other centers, postoperative examinations of especially the patients who had surgery were not present. Thirdly, a complete spinal scan could not be performed to detect other concomitant vertebral anomalies in the patients with fusion that was detected with cervical MSCT.

\section{Conclusion}

Congenital anomalies at the CVJ region, although rare, can lead to morbidity and mortality secondary to the compression to the vital structures at the CVJ region due to degenerative changes in the bone structure and dislocations in the joints with advancing ages. Therefore, MSCT and MRI studies on the CVJ region should be evaluated carefully at every age and the detected pathologies should be followed due to the risk of complications at advancing ages.

\section{References}

1. Pang D, Thompson DN. Embryology and bony malformations of the craniovertebral junction.Childs NervSyst 2011 Apr;27(4):523-564.

2.da Silva OT, Ghizoni E, Tedeschi H, Joaquim AF. Role of dynamic computed tomography scans in patients with congenital craniovertebral junction malformations.World J Orthop 2017 Mar 18;8(3):271-277.

3. Dickman CA, Lekovic GP. Biomechanical considerations for stabilization of the craniovertebral junction.Clin Neurosurg 2005;52:205-213.

4. Lustrin ES, Karakas SP, Ortiz AO, Cinnamon J, et al. Pediatric cervical spine: normal anatomy, variants, andtrauma. Radiographics 2003 May-Jun;23(3):539-560.

5. Offiah CE, Day E. The craniocervical junction: embryology, anatomy, biomechanics and imaging in blunt trauma. In sights Imaging 2017 Feb;8(1):29-47.

6. Menezes AH. Craniocervical developmental anatomy and its implications. Childs Nerv Syst 2008 Oct;24(10):1109-1122.

7. Pang D, Thompson DN. Embryology, classification, and surgical management of bony malformations of the craniovertebral junction. Adv Tech Stand Neurosurg 2014;40:19-109.

8. Sharma DK, Sharma D, Sharma V. Atlantooccipital Fusion: Prevalence and its Developmental and Clinical Correlation. J Clin Diagn Res 2017 Jun;11(6):AC01-AC03.

9. Khamanarong K, Woraputtaporn W, Ratanasuwan S, Namking M, Chaijaroonkhanarak W, SaeJungS.Occipitalization of the atlas: its incidence and clinical implications. Acta Med Acad 2013;42(1):41-45. 
10. Mubarak AI, Morani AC. Anomalous vertebral arteries in Klippel-Feil syndrome with occipitalized atlas: CT angiography. Radiol Case Rep 2018 Feb 22;13(2):434-436.

11. Gruber J, Saleh A, Bakhsh W, Rubery PT, MesfinA.The Prevalence of Klippel-Feil Syndrome: A Computed Tomography-Based Analysis of 2,917 Patients. Spine Deform 2018 Jul - Aug;6(4):448-453.

12. Jumah F, Alkhdour S, Mansour S et al. Os Odontoideum: A Comprehensive Clinical and Surgical Review. Cureus 2017 Aug 8;9(8):e1551.

13. Jain N, Verma R, Garga UC, Baruah BP, Jain SK, Bhaskar SN. CT and MR imaging of odontoid abnormalities: A pictorial review. Indian J Radiol Imaging 2016 Jan-Mar;26(1):108-119.

14. Smoker WR. Craniovertebral junction: Normal anatomy, craniometry, and congenital anomalies. Radiographics 1994;14:255-277.

15. Goel A, Bhatjiwale M, Desai K. Basilar invagiation: A study based on 190 surgically treated patients. J. Neurosurg 1998;88:962-968.

16. Basaran R, Efendioglu M, Senol M, Ozdogan S, Isik N. Morphometric analysis of posterior fossa and craniovertebral junction in subtypes of Chiari malformation.Clin Neurol Neurosurg 2018 Jun;169:1-11.

17. Ferreira JA, Botelho RV. The odontoid process invagination in normal subjects, Chiari malformation and Basilar invagination patients: Pathophysiologic correlations with angular craniometry. Surg Neurol Int 2015 Jul 8;6:118.

18. Kumar R, Kalra SK, MahapatraAK. A clinical scoring system for neurological assessment of high cervical myelopathy: measurements in pediatric patients with congenital atlanto-axial dislocations.Neurosurgery 2007 Nov;61(5):987-993.

19. Kumar R, Nayak SR. Management of pediatric congenital atlantoaxial dislocation: A report of 23 cases from northern India. Pediatr Neurosurg 2002 Apr;36(4):197-208.

20. Yang SY, Boniello AJ, Poorman CE, Chang AL, Wang S, Passias PG. A review of the diagnosis and treatment of atlanto-axial dislocations. Global Spine J 2014 Aug;4(3):197-210. 